Industrial Engineering

\& Management Systems

Vol 12, No 4, December 2013, pp.289-305

http://dx.doi.org/10.7232/iems.2013.12.4.289

ISSN 1598-7248 | EISSN 2234-6473 |

(C) 2013 KIIE

\title{
A Review on Yard Management in Container Terminals
}

\author{
Lu Zhen* \\ School of Management, Shanghai University, Shanghai, China \\ Xinjia Jiang, Loo Hay Lee, Ek Peng Chew \\ Department of Industrial and System Engineering, National University of Singapore, Singapore
}

(Received: November 30, 2013 / Accepted: December 6, 2013)

\begin{abstract}
With advancements of quay side equipments and technologies, the bottleneck of port operations has moved from quay side to yard side. The yard management of a port has significant influences on the competitiveness of a port in the global shipping network. The research area of yard management has attracted a lot of attentions from both the academia and the industrial practitioners. This paper gives a comprehensive review for the studies on the yard management in container terminals. From three aspects, i.e., yard cranes management, yard vehicles management, and yard spaces management, this paper reports the advances in these three areas. Some future directions on the yard management researches are also discussed. The purpose of this paper is to stimulate more practically relevant researches in this emerging area.
\end{abstract}

Keywords: Port Operation, Container Terminals, Yard Management, Review

* Corresponding Author, E-mail: lzhen.sh@gmail.com

\section{INTRODUCTION}

Since 1990s, world container traffic has been growing at most three times world GDP growth, due to the offshoring of manufacturing operations in Asia, in particular China. Port throughputs increased even faster because an increasing number of containers are transshipped. Efficient port operations that maximize the throughput (ports are paid by a handling charge per container) are essential for port operators' profits. With advancements of quay side equipments and technologies (e.g., twin 40-ft quay cranes, indented berths, etc.), the bottleneck of the port operations has moved from quay side to yard side. The yard management of a port has significant influences on the competitiveness of the port in global shipping network.

Generally speaking, the containers handled in the storage yard can be categorized into three types, namely "import", "export" and "transshipment". For import, the containers arrive in large batches which are brought in by vessels. They will be temporarily stored in the yard until being retrieved by individual local customers. For export, the containers are brought in by the local customers and accumulated in the storage yard. When their destination vessel arrives, the export containers will be loaded together onto the vessel. For transshipment containers, the process is a little different. The containers will be temporarily stored in the yard after being brought in by a vessel. Instead of being retrieved by local customers, they will be eventually loaded onto other vessels and transported to their next destinations.

To efficiently perform these container handling activities, the storage yard management mainly considers three kinds of yard resources, namely transport vehicles, yard cranes and the storage space (Figure 1). Due to the limited land, containers in the yard are usually stacked in multi-level blocks. The storage space is managed as many blocks, which can be shown with the example in 


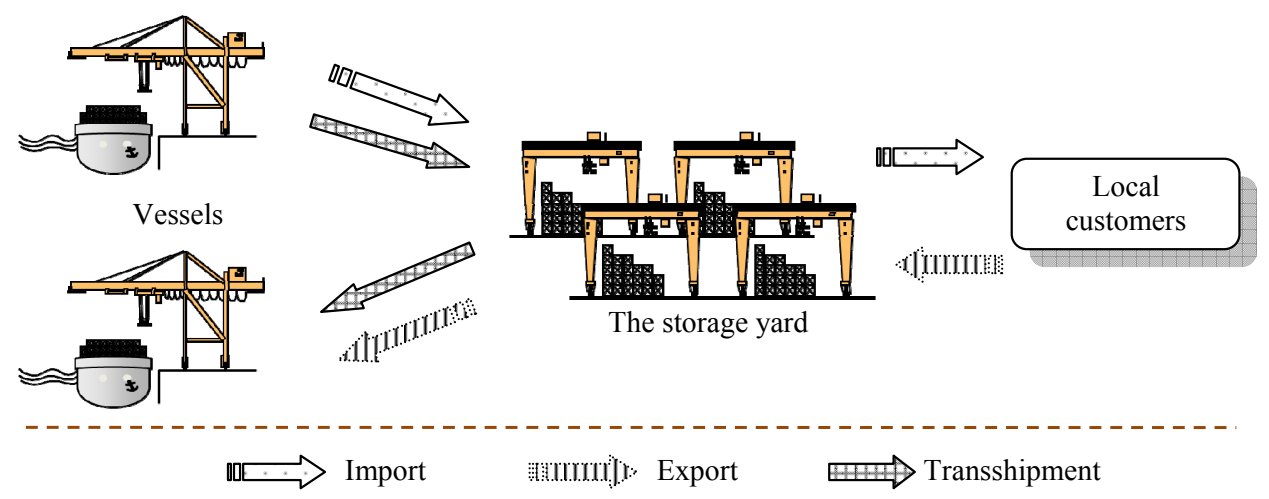

Figure 1. Container handling activities in the storage yard.

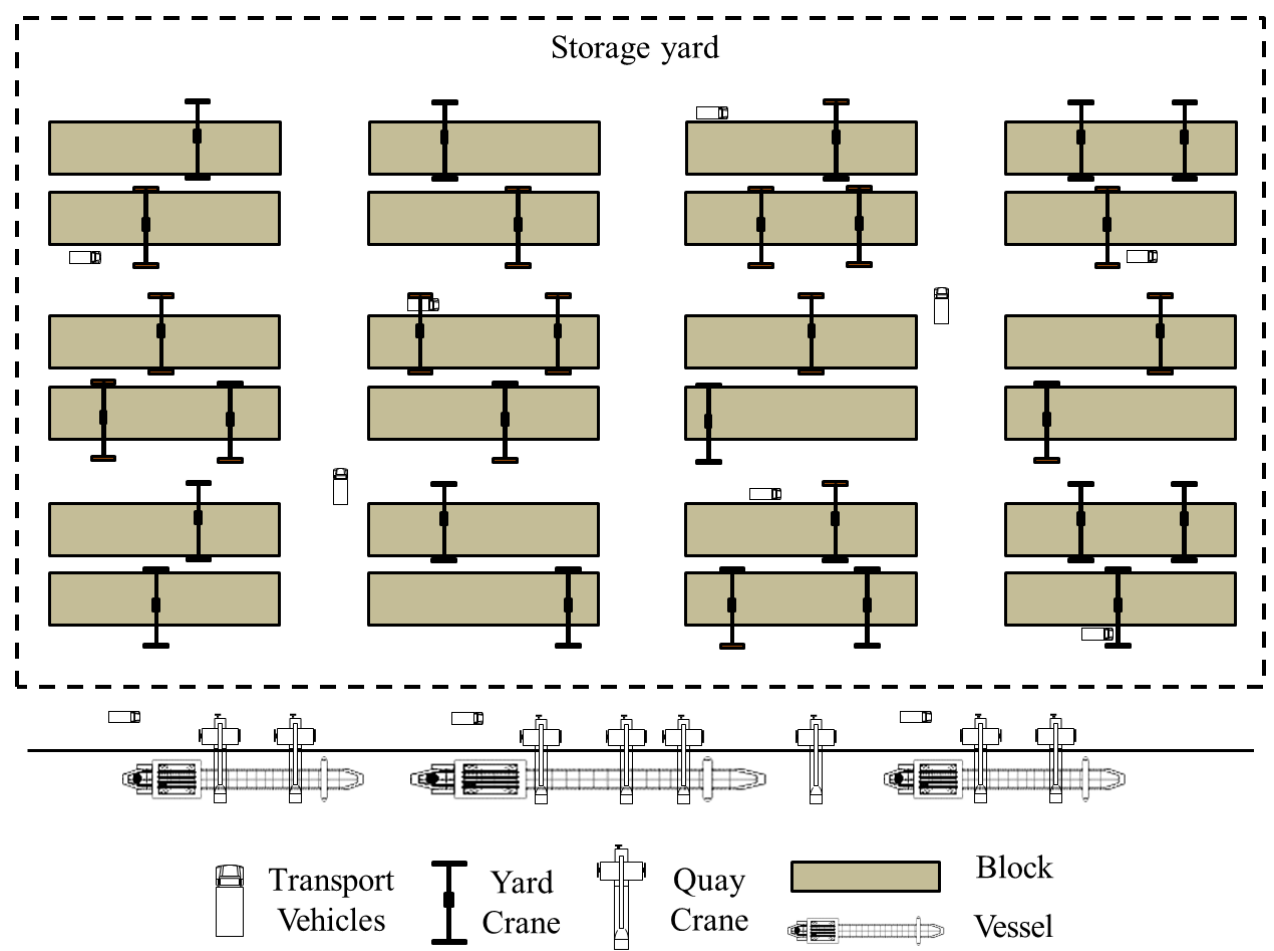

Figure 2. General picture of the storage yard.

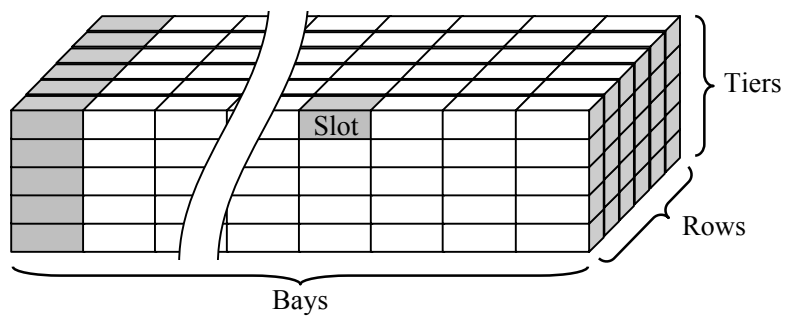

Figure 3. Container stacking in a block.

Figure 2. Within each block, the containers are stacked on top of another by the yard cranes. As shown in Figure 3, a typical block can be described in three dimensions, namely "bay", "row" and "tier." The configuration of a block depends on the yard cranes used for con- tainer stacking. The basic unit of the storage space is "slot", which can fit one TEU (20-foot equivalent unit). As the container stacking is carried out by yard cranes, the specific configuration and layout of yard blocks depend on yard cranes. The delivery of containers is carried out by transport vehicles.

Based on these resources, the storage yard management can be divided into three decision levels, namely "strategical level", "planning level" and "operational level" as shown in Figure 4. The strategical level is considered at the construction or initial phase of the storage yard. The decisions at this level mainly include the storage strategy and the equipment mix (types and number of yard cranes and transporting vehicles to be equipped in the yard). Few research works can be found 


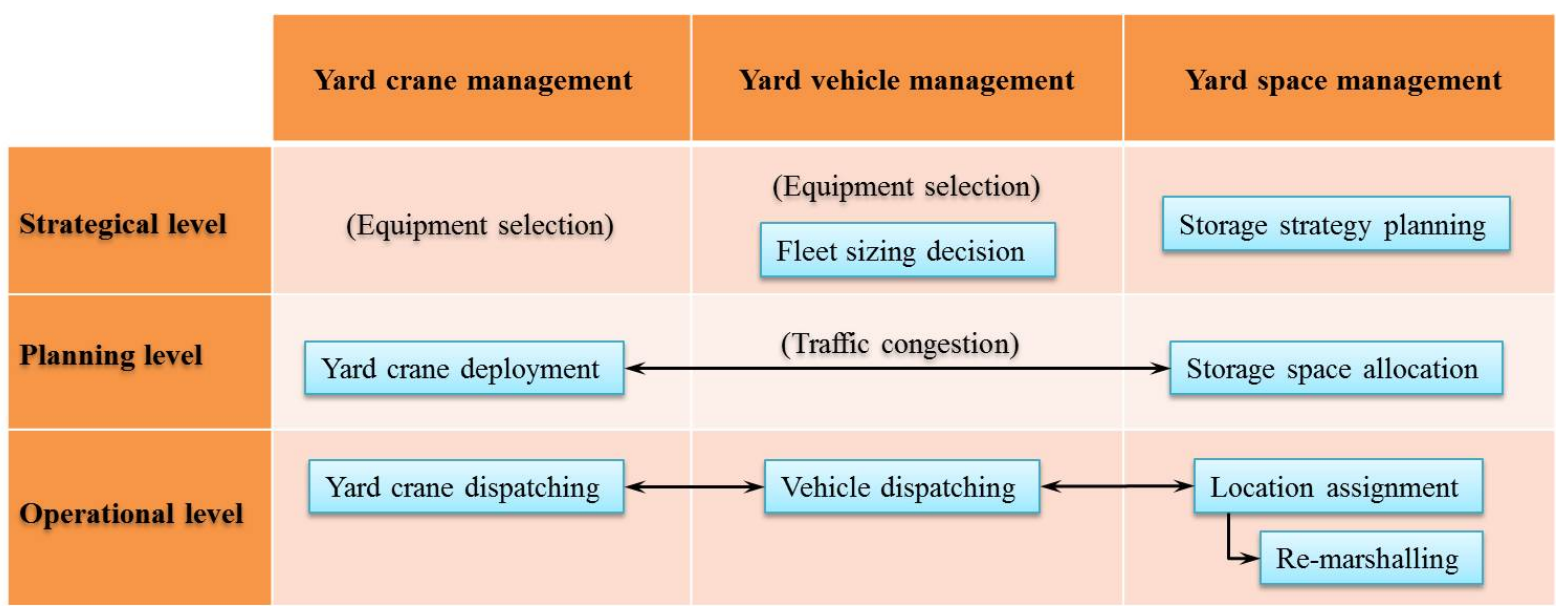

Figure 4. Major research areas on storage yard management.

focusing on equipment selection, but they are the basis of the main research topics at this level, such as the storage strategy planning and fleet sizing decision. The planning level decides how to allocate storage space to incoming containers and how to deploy yard cranes for container handling. To ensure the traffic flow of containers, the traffic congestion of transporting vehicles is generally considered in these two research topics. At the operational level, the decisions for all yard resources are detailed to each container. At this level, the interactions among the yard crane, the transporting vehicle and the storage location of each container have to be considered carefully to improve the operation efficiency. Research works can be found focusing on each type of yard resource, as well as integrating all yard resources. As the storage location at the operational level directly affects the yard crane dispatching and vehicle dispatching, many studies can be found focusing on "re-marshalling" which is usually performed in the container retrieval process. Following this structure, out paper reviews the studies on the yard management in container terminals through three aspects, i.e., yard cranes management, yard vehicles management, and yard spaces management. Some future directions on the yard management researches are also discussed at the end of this paper.

\section{YARD CRANE MANAGEMENT}

The yard cranes are the crucial equipment in the storage yard to perform the storage and retrieval of containers. The yard crane management can be divided into two levels, namely the yard crane dispatching and the yard crane deployment. For the yard crane dispatching problem, the main purpose is to decide the route of the yard cranes within a block. For the yard crane deployment problem, the main concern is the number of yard cranes to be deployed in each block and how to shift the yard cranes among the blocks.

\subsection{Yard Crane Dispatching}

When there is only one yard crane under consideration, the dispatching problem is to decide the number of containers to pick up in each bay as well as the sequence of the yard bays that the yard crane visits. Kim KH and Kim KY (1999b) considered the dispatching of a single yard crane, with a given load plan and a given bay plan for export containers. The number of containers to pick up was formulated as a transportation problem, while the visiting route was determined with a dynamic programming procedure. Narasimhan and Palekar (2002) analyzed the generalized problem of scheduling yard cranes to pick up the containers. They did a theoretical investigation of the structural properties of the problem. Based on the results of the investigation, the problem was proved to be NP-complete and formulated as an integer program. Both exact and heuristic algorithms were developed to solve the problem. Heuristics for the same problem was studied in Kim and Kim (2003), where a genetic algorithm and a beam search algorithm were developed for the dispatching of a single yard crane for loading jobs. The numerical experiments showed that both algorithms can find near optimum solutions for small problems of ten yard bays. However, the neiborhood beam search algorithm outperformed the genetic algorithm for large scale problems. Kim et al. (2003) studied the delivery and receiving operations for a single yard crane in order to reduce the total delay time of external trucks. Different sequencing rules were compared with the dynamic programming approach. In previous studies, the handling sequence of an individual container was not decided. Kim et al. (2004) took this into consideration and solved the whole problem in two sub-problems. The first sub-problem decided the travel route of a yard crane as well as the number of containers to pick up at each bay. The second sub-problem decided the load sequence for individual containers. A beam search algorithm was developed to combine these two 
sub-problems. Ng and Mark (2005a, 2005b) studied the scheduling of a single yard crane for a given set of loading and unloading containers with different ready times. Efficient heuristics were provided to find the lower bounds and upper bounds, while a branch and bound algorithm was proposed to solve the NP-complete problem. The numerical experiments showed that the optimal handling sequence for individual containers can be found for most instances.

When more than two yard cranes are studied at the same time, the cooperation of the yard cranes to serve the common quay crane and the interference among the yard cranes shall be considered. Zyngiridis (2005) used integer linear programming to study the scheduling of one or two RMGs of equal size in a single block. The data from Rotterdam with different characteristics was used in the numerical experiment to evaluate the performance of the RMGs. It was found that the performance of single RMG was significantly affected by the block size and the fill level of each block, while the performance of two RGMs was only affected by the length of the block. Besides, the case of two RMGs was always better than the case of one RMG in terms of efficiency. Cao et al. (2006a) discussed the scheduling of yard cranes considering the loading sequence requirements. In their problem, two yard canes were deployed to serve loading containers to the same quay crane. The two yard cranes did not interfere with each other, as they picked up containers in separate blocks. A revised genetic algorithm was proposed to find the solutions. A variation of the problem was studied in Cao et al. (2006b) for a system with multiple yard cranes. An algorithm based on the simulated annealing and a greedy heuristic were developed to find the scheduling sequence of yard cranes to minimize the total handling time. The performance of the algorithms was tested with randomly generated experiments. It was shown that the greedy heuristic can find outperforming solutions compared with the simulated annealing algorithm in limited computation time. $\mathrm{Ng}$ (2005) studied the problem of scheduling multiple yard cranes which shared the single bi-directional travel lane and cannot pass through each other. The scheduling problem was formulated as an integer programming model to minimize the total loading time or the sum of truck waiting times. A heuristic based on dynamic programming was developed to solve the problem, and an algorithm was provided to find the lower bound. Jung and Kim (2006) studied the problem of scheduling multiple yard cranes which served multiple quay cranes, where the adjacent yard cranes working in the same block had interference with each other. The algorithms based on genetic algorithm and simulated annealing approaches were proposed to schedule the travelling route of the yard cranes and number of containers to pick up in each yard bay. Jung et al. (2006) extended the problem to schedule the loading sequence of the quay cranes considering the interference of multiple yard cranes. A greedy randomized adaptive search procedure was pro- posed for constructing a schedule to minimize the makespan of the quay cranes. The numerical experiments showed that the improvement phase of the heuristic search algorithm was too time consuming for practice. Lee et al. (2007) studied the problem of scheduling two yard cranes with the simulated annealing algorithm. The two yard cranes travelled in separate blocks and serve the same quay crane for loading operation. The main objective was to minimize the total loading time at the stack area. The numerical experiments showed that the proposed algorithm can find solutions close to the lower bound. Chang et al. (2011) studied the scheduling of yard cranes in a rolling horizon approach. The yard cranes were deployed in separate zones to avoid interference during operation. Due to the computational scale of the problem, a heuristic algorithm integrated with a simulation model was proposed to generate the initial solutions, which were further optimized with a genetic algorithm.

Some studies have also addressed especially for the crossover rail mounted rail mounted gantry crane (RMG) system. For this kind of system, two or more RMGs are deployed in the same block. Since the RMGs are of different size, they can pass through each other without blocking the way. This system was studied in Cao et al. (2008), Dorndorf and Schneider (2010), and Vis and Carlo (2010).

\subsection{Yard Crane Deployment}

Due to the vessel schedule and the storage strategies used for container assignment, the distribution of workload among the blocks changed over time. As the yard cranes are very expensive equipment, the port operators usually do not keep a fixed number of yard cranes in each block. Instead, the yard cranes are deployed dynamically among the blocks to fit the changing workload distribution and finish the workload within the planned time periods. Zhang et al. (2002) studied the yard crane deployment to decide the deployment times and routes of the yard cranes, based on the given workload distribution in each period during the planning horizon. The problem was formulated as a mixed integer program and solved with lagrangian relaxation techniques. Cheung et al. (2002) analyzed the computational complexity of the problem. Besides a Lagrangian decomposition solution procedure, a new solution approach called the successive piecewise-linear approximation method was proposed to solve the problem. The numerical experiments showed that their new solution approach was efficient and effective for large scale problems. The similar problem was also studied in Linn and Zhang (2003), and Linn et al. (2003). In previous studies, the workload was commonly estimated based on the number of containers to be handled in each period, which was often inaccurate in practice. Guo and Huang (2012) proposed a hierarchical scheme to divide the workload and deploy the yard cranes. A time partition plan divided the planning horizon into smaller time windows; while a space partitioning algorithm flexibly divided the 
workload into non-overlapping zones for yard crane deployment in each time window. The yard crane dispatching problem can be combined for the job sequence in each zone. However, these studies were all based on given or estimated workload distribution, which was determined by space allocation to incoming containers. Thus, many studies combined the yard crane deployment with the space allocation decisions.

\section{YARD VEHICLE MANAGEMENT}

\subsection{Vehicle Dispatching}

Vehicle dispatching is an operational level decision problem in yard management. It is to determine which vehicle transports which container. For conventional trucks and trailer systems, the vehicle dispatching problem is widely studied.

Bish (2003) and Bish et al. (2001, 2005) are the pioneers in the area of yard vehicle dispatching problems. Bish et al. (2001) studied a problem on assigning each discharged container to a yard location and dispatching vehicles to the containers so as to minimize the time it takes to discharge all the containers from the ship. They proved the problem is NP-hard and developed a heuristic algorithm based on formulating the problem as an assignment problem. Bish (2003) improved the above study by considering that each ship is served by multiple quay cranes, and proposed a problem on assigning each discharged container to a yard location, dispatching vehicles to the containers, and scheduling the loading and discharging operations on the cranes. The objective is also to minimize the makespan. They formulated the problem as a transshipment problem and proved the problem is NP-hard. In Bish et al. (2005), an extension of the problem in Bish (2003) was studied. They developed easily implementable heuristic algorithms for this problem and identified the absolute and asymptotic worst-case performance ratios of the proposed heuristics. Numerical experiments show that their proposed heuristics can generate near-optimal or optimal solutions for simple or general setting scenarios.

For the vehicle dispatching problem, job sequencing is a core decision in some studies. $\mathrm{Ng}$ and Mak (2004) studied how to sequence trucks for entering the working lanes so as to reduce the traffic congestions in the working lane. The objective is to minimize the total time required to serve all the empty trucks dispatched to a loading yard block. Ng et al. (2007) studied a problem of scheduling a fleet of trucks to perform a set of transportation jobs with sequence-dependent processing times and different ready times. The job sequencing decision can also be optimized with the job timing decision. For example, Zhang et al. (2005) studied a yard truck scheduling problem, in which the starting times as well as the order of vehicles for carrying out these jobs need to be determined. They proposed three mixed integer programming models and compared them by experiments, which showed that one model is the best among the three models and the greedy algorithm is capable of solving large scale problems. Nishimura et al. (2005) addressed a trailer routing problem at ports, where yard trailers are assigned to specific quay cranes. They designed an efficient trailer assignment method called 'dynamic routing', which can reduce travel distance and generate substantial savings in the trailer fleet size and overall cost by $15 \%$. Currently, traditional port operations problems are extended to a context of multiple terminals. He et al. (2013) studied a problem on scheduling trucks among multiple container terminals. An integer programming model was developed based on the rolling-horizon approach. Due to computational complexity, a genetic algorithm based simulation optimization method was developed. The genetic algorithm technique was applied to search the solution spaces, while the simulation module was employed to evaluate the solutions, repair the infeasible schedules, and execute the rolling-horizon approach.

The commonly used objective for the vehicle dispatching problem is the minimization of the transportation time. Narasimhan and Palekar (2002) studied a problem of minimizing the time for trucks to transport the containers from the yard onto the ship. They proved that the problem is NP-complete, and proposed a branch-andbound based enumerative method to obtain an exact solution to the problem. Li and Vairaktarakis (2004) investigate the problem of optimizing the time for trucks to transport containers between a ship and the yard. An optimal algorithm and some efficient heuristics were developed to solve the problem. The effectiveness of the heuristics was studied both analytically and computationally.

The vehicle dispatching decision problem can be integrated with other decision problems.

(1) Integrated with yard cranes scheduling: Cao et al. (2010) studied another integrated problem on yard truck and yard crane scheduling problems for loading operations in container terminal. The problem is formulated as a mixed-integer programming model. Two solution methods based on the benders cut were developed for solving the problem. Experiments were conducted to evaluate the effectiveness of the proposed solution methods.

(2) Integrated with storage allocation: Lee et al. (2009) studied an integrated optimization problem on the yard truck scheduling and the storage allocation. The objective is to minimize the weighted sum of total delay of requests and the total travel time of yard trucks. Due to the intractability of the proposed problem, a hybrid insertion algorithm was designed for effective problem solutions. Computational experiments were conducted to examine the key factors of the problem and the performance of the proposed heuristic algorithm. Wu et al. (2013) proposed an integrated optimization problem for storage management and vehicle scheduling at container 
terminals. A linear mixed integer programming model was proposed. They also investigated a nonlinear mixed integer programming model to reduce the number of constraints and the computational time. They proposed a genetic algorithm for the model to illustrate how large scale problems can be solved and to show the effect of different factors on the performances of the optimization model.

(3) Integrated with yard cranes and quay cranes scheduling: Chen et al. (2007) addressed an integrated model for scheduling various kinds of container handling equipments, i.e., yard trucks, yard cranes, and quay cranes. The integrated dispatching model was formulated as a hybrid flow shop scheduling problem with precedence and blocking constraints. A tabu search based algorithm was developed to solve the problem. Two heuristics for generating initial solutions were also investigated by numerical experiments. Chen et al. (2013) developed a constraint programming model for scheduling the cranes and the horizontal truck transportation simultaneously. They designed a three-stage algorithm to solve medium and large sized problems. The method was proved to be better than their previously proposed tabu search method. (4) Integrated with storage allocation and quay crane scheduling: Xue et al. (2013) proposed a framework for optimizating the yard truck dispatching, the yard location assignment, and quay crane scheduling. They took account of the loading and discharging precedence relationships between containers in the quay crane operations. In the first stage, an ant colony optimization algorithm was employed to generate the yard location assignment for discharging containers. In the second stage, the integration of the yard truck scheduling and the quay crane scheduling was formulated as a flexible job shop problem, and an efficient greedy algorithm and a local search algorithm were proposed.

Besides the traditional yard trucks (prime movers), some automated yard vehicles become more and more popular in container ports, especially some automated container terminals. The automated guided vehicles (AGVs) and the automated lifting vehicles (ALVs) are the two main types of the automated yard vehicles. Some other types of automated yard vehicles such as the straddle carriers, intelligent autonomous vehicles (IAVs) actually belong to some sort of ALV to some extent. The difference between the AGVs and the ALVs lies in that the latter one can lift containers from the ground by itself. The following two subsections 3.1.1 and 3.1.2 review the related works on these two types of automated yard vehicles, respectively.

\subsubsection{AGV dispatching}

AGVs are now becoming popular in automated container terminal systems at seaports. Its performance affects the efficiency of the entire system. As a large number of AGVs use the same infrastructural facilities, the control of AGV traffic at a container terminal is crucial to the system performance.
Some pioneering studies on AGV scheduling include: Evers and Koppers (1996) developed a formal tool to control a large number of AGVs. The control was imposed by using a hierarchical system called semaphores. Simulation models were built to evaluate the various dispatching rules. Kim and Bae (1999) studied how to assign tasks of container delivery to AGVs during ship operations in an automated container terminal. They assumed a dual-cycle operation, in which the loading and the discharging operation can be performed alternately. A mixed integer linear programming model was formulated with minimizing the completion time of all the discharging/loading operations at quayside, and the total travel time of AGVs. Kim and Bae (2004) studied how to dispatch AGVs by utilizing information about AGVs' locations and times of future delivery tasks. A mixed-integer programming model was developed for assigning delivery tasks to the AGVs. A heuristic algorithm was suggested for solving the model. A simulation study was also performed by considering the uncertainties of various operation times and the number of future delivery tasks for looking ahead. Grunow et al. (2004) studied a dispatching problem for multi-load AGV, which can carry more than one container at a time. A flexible priority rule based approach and a mixed integer linear programming model were developed. The performance of the priority rule and the model were analyzed for several scenarios with respect to total lateness of the AGVs. Grunow et al. (2006) presented a simulation study of AGV dispatching strategies in an automated container terminal. The dual load mode was used in the study. The performance of the proposed dispatching strategies was evaluated by a scalable simulation model.

AGV system layout and AGVs deployment have influence on the AGV systems' performance. Some typical studies are as follows. Gademann and van de Velde (2000) studied two AGV systems, one is the unidirectional flow system where AGVs can move in one direction only, and the other is the bidirectional flow system where AGVs can move in both directions. They analyzed the complexity of the algorithms for the problems. Their study showed that the deployment of AGVS may not be effective if the container terminal's layout is not good. Liu et al. (2004) developed some simulation models to investigate the impact of layout on performance of terminals that use AGVs. In particular, two terminals with different but commonly used yard configurations were compared. A multi-attribute decision making method was used to assess the performance and determine the optimal number of AGVs that should be deployed. Hoshino et al. (2007) used queuing network theory and developed a transportation simulator to analyze and compare the performance of the vertical and the horizontal AGV systems. Their analytical results showed that the horizontal AGV system is more effective than the vertical AGV system under most demand scenarios. 
Some AGV scheduling strategies are developed by borrowing ideas from other fields.

(1) Auction mechanism: Lim et al. (2003) suggested using an auction algorithm for the AGV dispatching problem. Different from traditional dispatching rules, the proposed dispatching rule looks into the future for an efficient assignment of delivery multiple tasks to multiple vehicles. The dispatching decisions were made through communication among related vehicles and machines. A simulation study was also conducted for comparing the performance of the method with that of a popular dispatching rule.

(2) Inventory control policy: Briskorn et al. (2006) investigated a real-time scheduling problem for assigning transportation jobs to AGVs. They presented an inventory-based formulation for the assignment problem to avoid the estimation of driving times, completion times, and tardiness because such estimates were often highly unreliable in practice. The model was solved using an exact algorithm. Simulation experiments were performed and showed that the inventory-based model had better performance than the commonly used due-time-based method.

(3) Artificial immune system: Lau et al. (2007) employed the methodology of artificial immune system (AIS) and proposed an immunity-based control framework, which had the ability to detect changes, adapt to dynamic environment and coordinate vehicles activities for goals achievement. Their proposed system can help port operators deploy a fleet of autonomous guided vehicles for material handling in an automated yard.

(4) Real-time systems: Angeloudis and Bell (2010) developed a new dispatching approach for real-time control of AGVs in container terminal settings under various conditions of uncertainty. Several performance indicators were presented, focused on generic features of vehicle operations as well the assessment of uncertainty levels inside the terminal. Their simulation experiments showed that the proposed technique outperformed some well known heuristics and alternative algorithms.

Deadlock and conflict resolution Deadlock is a serious problem in AGV systems. Deadlock is a situation in which at least a part of the AGV system stalls. There are a lot of situations in which the system may stall and most of these situations can be prevented by a good control and navigation system. There existed a variety of deadlock-detecting algorithms for manufacturing systems, in which the network layout was simple and the number of AGVs is small. Therefore, some scholars investigated methods on AGV deadlock prevention in automated container terminals. In reality, AGVs are liable to deadlocks as they always need a secondary resource, either a quay crane or a stacking crane, to perform the pick-up and drop-off operations. Due to the absence of container buffering between AGVs and cranes, the consequences of deadlocks are rather severe in reality.

Rajeeva et al. (2003) proposed an efficient AGV deadlock prediction and avoidance algorithm for a largescale container terminal, which had complex layout and involved about 80 AGVs. The proposed solution was implemented by the AutoMod simulation software. Their results showed that all the potential deadlock situations can be detected and avoided via this methodology. Lehmann et al. (2006) investigated the deadlock handling of AGVs for an automated terminal. Two methods to detect the deadlock were discussed. One is based on the matrix representation of the terminal system. The other directly traces the requests for each individual resource. Kim et al. (2006) developed an efficient deadlock prediction and prevention algorithm for AGV systems. Because an AGV may occupy more than one grid-block at a time, they proposed a method for reserving grid-blocks in advance so as to prevent deadlocks. A graphical representation method and a priority table were suggested for maintaining priority consistency among grid-blocks. Reveliotis (2000) employed a concept of zone control and proposed a conflict resolution strategy for conflictfree routing in AGV systems. The zone control determines vehicle routes incrementally, one zone at a time. Their proposed strategy can ensure both the robust AGV conflict resolution and the operational flexibility, which means that vehicles can travel freely on arbitrarily structured guide path networks.

\subsubsection{ALV, SC and IAV dispatching}

Besides the AGVs, automated container terminal systems also use some other automated yard vehicles. Recently, a lot of studies have been performed to investigate the performance of these new resource systems.

ALV is capable of lifting a container from the ground by itself. If a port uses AGVs, there exists significant unproductive and costly waiting both under the yard cranes and in the blocks. A possible alternative solution to this problem is the use of ALVs, which can load and unload their own containers. Nguyen and Kim (2009) studied how to dispatch ALVs by utilizing information about pickup and delivery locations and time in future delivery tasks. A mixed integer programming model was formulated for assigning transportation tasks to ALVs. Numerical experiments were conducted to analyze the effects of dual cycle operation, number of ALVs, and buffer capacity on the performance of ALVs.

Straddle carriers (SC) are alternative vehicles for the transport, retrieval and storage of containers. Thus the routing of straddle carriers has received much attention from the researchers. In Steenken (1992) the routing problem of straddle carriers was studied. The problem was formulated as a linear assignment problem with the objective of minimizing the empty travel distance by combining loading and unloading jobs. As a result, a saving of $13 \%$ in the empty travel distance was obtained. In Steenken et al. (1993), different methods for the routing of the straddle carriers were tested to minimize no- 
load ways. Heuristics solving the Multiple Travelling Salesman Problem were applied to the routing problem as well as a method for sequencing insertions in printed circuit board assemblies and rules for machine scheduling. Simulation with real data indicated a total saving of about 20,000 km a year. Kim KH and Kim KY (1999b) studied the single straddle carrier routing problem to load export containers onto a containership. An integer programming model was developed with the objective of minimizing the total traveling time of the straddle carrier. Kim KH and Kim KY (1999a) studied the straddle carrier routing problem during the loading operation of export containers. The objective was to minimize the total traveling distance of all the straddle carriers in the storage yard. A beam search algorithm was developed to solve the straddle carrier routing problem. Numerical experiments were carried out to evaluate the proposed algorithm.

IAVs: Gelareh et al. (2013) studied a new class of yard vehicles, i.e., IAVs, which is technologically superior to the existing AGVs in many respects. In order to accommodate this new feature of IAVs, their study extended one of the existing mixed integer programming models of AGV scheduling in order to minimize the makespan of operations for transporting a set of containers of different sizes between quay cranes and yard cranes. They also developed a Lagrangian relaxation based decomposition approach equipped with a primal heuristics to solve the problem.

Comparison among ALV and AGV systems: Yang et al. (2004) developed a simulation model of an ACT with perpendicular layout for comparing the productivity of the ALVs and the AGVs in ACTs. From the results of the simulation analysis, they investigated the saving effect with respect to the cycle time and the required number of vehicles. Their study demonstrated that the ALV was superior to the AGV in both productivity and efficiency principally because the ALV can eliminate the waiting time in the buffer zone. Vis and Harika (2004) also used simulation method to compare the productivities of AGVs based system and the ALVs based system. The comparative criteria included unloading times of a ship, occupancy degrees and the number of vehicles required. Their study found that about $38 \%$ more AGVs need to be used than ALVs. Their study concluded that ALVs are a cheaper option than AGVs. In addition, their study also indicated that the design of the terminal and technical aspects of quay cranes influenced the number of vehicles required. Bae et al. (2011) also used simulation method to compare the operational productivities of the AGVs and ALVs. Their simulation model considered traffic control scheme and assumed a flexible path layout, in which the vehicles can move almost freely in any vertical and horizontal directions. The results showed that the ALVs reached the same productivity level as the AGVs using much less number of vehicles due to its self-lifting capability. However, the results also revealed that the AGVs eventually catch up the performance of the ALVs in most cases if a sufficient number of vehicles are available. Another interesting finding was that when the tandem double trolley quay cranes were used for loading, the AGVs cannot catch up the ALVs no matter how many more vehicles are added. Duinkerken et al. (2006) compared three transportation systems (multi-trailers, AGVs, and ALVs) for the overland transport of containers between container terminals by using a simulation model, which was equipped with a rulebased control system as well as an advanced planning algorithm. Their study provided some insights into the importance of the different characteristics of the transport systems and their interaction with the handling equipment. In addition, a cost analysis was also executed to support management investment decisions.

\subsection{Fleet Sizing Decision}

For port container terminals, one of the decisions is the determination of the necessary number of transport vehicles. Steenken (1992) formulated a linear assignment model to determine the fleet size of straddle carriers in a container terminal. Vis et al. (2001) developed a model and a strongly polynomial time algorithm (minimum flow algorithm) to determine the necessary number of AGVs required in a semi-automated container terminal. Koo et al. (2004) investigated the fleet size problem to determine the necessary number of vehicles required to handle the containers. They proposed a tabu search algorithm aimed at simultaneously finding the minimum fleet size required and travel route for each vehicle while satisfying all the transportation requirements within the planning horizon.

\section{YARD SPACE MANAGEMENT}

\subsection{Storage Strategy Planning}

The storage strategies applied in the yard depends on the type of containers. Generally speaking, the storage strategy is chosen to best utilize the relative fixed information of incoming containers, so as to store them in the proper location to facilitate container retrievals. For import containers, they are usually discharged from vessels in larges batches and retrieved by individual customers in small numbers. The retrieval time is unknown, but is often related to the arrival time. Thus, the segregation strategy is popular among the studies to separate the new incoming containers from the old ones to facilitate the retrieval. For export containers, their arrival time is often unknown but the retrieval time is relatively fixed by the destination vessels. Besides, the loading sequence of export containers may depends on other information, such as weight. Thus, clustering strategy is 
Table 1. Popular storage strategies in different containers

\begin{tabular}{|c|c|c|c|}
\hline \multicolumn{2}{|c|}{ Container terminal } & $\begin{array}{c}\text { Storage } \\
\text { strategies }\end{array}$ & Research works \\
\hline \multirow{3}{*}{$\begin{array}{c}\text { Gateway } \\
\text { ports }\end{array}$} & $\begin{array}{l}\text { Import } \\
\text { containers }\end{array}$ & $\begin{array}{l}\text { Segregation } \\
\text { strategy }\end{array}$ & $\begin{array}{l}\text { Castilho and Daganzo (1993) } \\
\text { Kim and Kim (1999) } \\
\text { Kim and Kim (2002) } \\
\text { Sauri and Martin (2011) }\end{array}$ \\
\hline & & & $\cdots$ \\
\hline & $\begin{array}{c}\text { Export } \\
\text { containers }\end{array}$ & $\begin{array}{l}\text { Clustering } \\
\text { strategy }\end{array}$ & $\begin{array}{l}\text { Taleb-Ibrahimi et al. (1993) } \\
\text { Kim and Park (2003) } \\
\text { Dekker et al. }(2006) \\
\text { Lim and Xu (2006) } \\
\ldots\end{array}$ \\
\hline \multicolumn{2}{|c|}{$\begin{array}{l}\text { Transshipment } \\
\text { ports }\end{array}$} & $\begin{array}{c}\text { Consignment } \\
\text { strategy }\end{array}$ & $\begin{array}{l}\text { Lee } \text { et al. }(2006) \\
\text { Han } \text { et al. }(2008) \\
\text { Zhen } \text { et al. }(2011) \\
\text { Jiang et al. }(2012,2013) \\
\text { Zhen }(2013) \\
\ldots\end{array}$ \\
\hline
\end{tabular}

popular among the existing studies to group the containers according to the information that affects the retrieval time. For transshipment containers, both arrival and retrieval time are relatively fixed. The consignment strategy is usually applied to store containers according to their destination vessels. Typical studies addressing each storage strategies can be categorized in the following table. Some of them will be further discussed in space allocation section.

For import containers, Castilho and Daganzo (1993) analyzed the segregation and non-segregation strategies with a simulation study. The first strategy separated the new incoming containers from the old ones to facilitate the retrieval, while the second strategy mixed the containers. An idealized case was used to identify the conditions favoring each strategy. It was found that the segregation strategy can reduce reshuffles for import containers when the retrieval sequence was affected by the arrival time. Sauri and Martin (2011) followed the research line of Castilho and Daganzo (1993) to further develop the segregation and non-segregation concepts. Three storage strategies were proposed for import containers, regarding how to mix the containers arriving in different batches. A mathematical model based on probabilistic distribution functions was developed to evaluate the reshuffles. The study showed that the choosing of proper strategy depends on the stacking height, the inter arrival time of vessels, and the dwell time of containers.

For export containers, Dekker et al. (2006) used detailed simulation experiments to test the different stacking policies in automated container terminals. It was found that category stacking for export containers can lower the number of reshuffles. Usually, the containers to the same destination vessel were stored together to facilitate the loading process, which shall be called the "clustering strategy." Taleb-Ibrahimi et al. (1993) poin- ted out that almost $50 \%$ of the space reserved was empty waiting for future arrivals, if the space was only allocated for once. Thus, they proposed to store the early incoming containers in a temporary area, before the permanent area was allocated. The best time to allocate the permanent space was decided based on the arrival pattern of export containers. However, their strategy improved the space utilization at the cost of double handling.

The strategies above are all studied for gateway port, which did not sufficiently address the particular needs of transshipment hubs. In a gateway port, the loading and unloading activities can be considered independently by separating import and export containers. For transshipment hubs like the Singapore port, around $74 \%$ of the containers are transshipment, which are discharged from one vessel and loaded onto other vessels. To avoid double handling, a container will stay in the same location until being retrieved. The "consignment strategy" is generally applied in a transshipment port, which can be considered as a more advanced "clustering strategy." Under the consignment strategy, the whole storage yard is managed as small storage locations preserved for destination vessels. The reservation of storage locations for each destination vessel is called the "yard template." The yard template planning problem was first studied in Han et al. (2008) to avoid potential congestions and facilitate the yard crane deployment. An iterative improvement method was developed to solve the problem, in which a tabu search based heuristic algorithm was used to generate an initial yard template, and then the generated yard template was improved by an improvement algorithm iteratively until an optimal or a satisfactory solution was obtained. In Zhen et al. (2011) the yard template planning was combined with berth allocation to minimize the service cost that was incurred by the deviation from vessels' expected turnaround time intervals, and the operation cost that was related to the route length of transshipment container flows in yard. A mixed-integer programming model was proposed to integrate the berth allocation and the yard template planning, while a heuristic algorithm was developed for solving the problem in large-scale realistic environments. Zhen (2013) further studied the yard template planning problem with considering uncertain berthing time and berthing position. A meta-heuristic was also suggested to solve the model and obtain a robust yard template under uncertainty.

Although the consignment strategy facilitates a faster loading process, it is known to be inefficient in space utilization. However, the consignment strategy was known to be inefficient in space utilization since each storage location must be dedicated to a particular vessel. To improve the space utilization while retaining the advantage of consignment, new storage strategies were proposed, namely the "partial space-sharing strategy" and the "flexible space-sharing strategy." In the "partial spacesharing strategy" proposed by Jiang et al. (2012), part of 
the storage space was allowed to be shared between two adjacent storage locations. The space in each storage location is divided into non-sharing and sharing parts. When less space is needed by a storage location, the sharing space in this storage location can be lent to the adjacent locations. The sharing space will then be returned, before the major workload comes into this storage location. Since the major containers to each destination vessel arrive at different periods, the storage locations preserved for the vessels will also need the sharing space during different shifts. An integrated framework is developed to decide the yard template and the container assignment at the same time. A more advanced "flexible space-sharing strategy" was proposed by Jiang et al. (2013). Under this strategy, the same storage location is allowed to be reserved for two vessels. The amount of space will only be allocated to a specific vessel on the arrival of corresponding containers. By controlling where to stack the containers in the storage locations, the containers to each vessel are not mixed and the consignment feature can be preserved. This strategy is formulated as a mixed integer program and solved with an algorithm based on the block diagonal structure of the model.

\subsection{Storage Space Allocation}

The space allocation problem studies the short-term container assignments to blocks or bays, which decides the distribution of container handling activities in the yard as well as the deployment of yard resources. Under the segregation strategy for import containers, Kim and Kim (1999) studied the long-term space allocation and the yard crane requirement considering the arrival pattern of import containers. It was formulated as an assignment problem constrained by the space capacity, and solved using Lagrangian relaxation technique. Kim and Kim (2002) further explored the same strategy, where they studied how to optimize the space needed for the given container volume. Under the clustering strategy for export containers, Lim and $\mathrm{Xu}$ (2006) considered the long-term space allocation as a two-dimensional packing problem. The space to each cluster of containers was allocated according to the requirements of incoming containers at different time periods, instead of assigning all the space for once. Under the consignment strategy for transshipment containers, Lee et al. (2006) studied the space allocation to determine the long term yard crane deployment. A consignment strategy was used to store containers going to the same destination vessel together. A high-low workload balancing protocol was proposed to control the congestions under consignment. The main idea was to avoid high workload appearing in neighboring storage locations. A MIP model and solution approaches were developed for space allocation based on this idea. The long-term space allocation for the space-sharing strategies was combined with template planning in Jiang et al. $(2012,2013)$.

Despite the difference in storage strategies, the above studies all address the long-term problems where the incoming containers are given for each time period during the whole planning horizon. This is mainly because the space allocation in one period has impact on future period. However, during the short-term operation, the incoming containers are only known for a short period in advance. An effective solution is the rolling horizon method, which has been used in both Kim and Park (2003) and Zhang et al. (2003) for space allocation. The essence of the rolling horizon method is to plan based on the realized information of the current period and the estimation of the near future. After implementing the plan for the current period, a new plan will be formulated based on the latest realized and estimated information. In Kim and Park (2003), a space allocation method was developed for export containers within limited space. The main target was to minimize the travel distance between the apron and the storage location. To utilize the storage space more efficiently, the space allocation for one vessel is divided into small stages, to satisfy the space requirements at each stage during the planning horizon. The space is allocated in terms of bays, and the rolling horizon approach is used when implementing the model. Zhang et al. (2003) studied a storage space allocation problem by a rolling horizon approach, where the problem of each period is divided into two levels. The first level balances the workload assigned to each block, while the second level minimizes the transfer distance of containers. Various resources have been considered in the study, such as quay cranes, yard cranes, storage space and internal trucks. Bazzazi et al. (2009) studied an extension for the first level decisions of the space allocation problem in Zhang et al. (2003). The type of containers affected the assignment of containers to blocks. A genetic algorithm was used to solve the problem.

\subsection{Location Assignment}

When the containers are stacked in multi-levels, only those on top can be accessed directly. The extra moves to reposition the containers on top of a requested one are called "reshuffles." As they increase the retrieval time, it is important to reduce the reshuffles through proper container stacking. Sculli and Hui (1988) is one of the first to study the relationship between reshuffles and space utilization considering the stacking configuration. Watanabe (1991) and Kim (1997) then developed different methodologies to estimate the reshuffles in container retrieval, which enlighten the later studies. Chen (1999) discussed the main causes for reshuffles as well as the different methods that can be used to reduce the reshuffles.

One of the commonly used methods is to store the incoming containers at a proper location to minimize the expected reshuffles. Kim et al. (2000) proposed a methodology to determine the storage location of arriving export containers considering the weight. It was assumed that heavy containers will be loaded before the light 
ones to keep the stability of the vessel. Thus, putting heavier ones on top will reduce the reshuffles. A dynamic programming model was formulated to find the optimal solution. As solving the model directly was time consuming, a decision tree was developed to support the real-time decisions. Kang et al. (2006) studied the storage location under the similar set, but the weight information of each arriving container was only an estimate. A simulated annealing approach was proposed to derive a good stacking strategy with uncertain weight information. Simulation experiments showed that the derived strategies were more efficient in reducing the reshuffles compared with the traditional same-weight-group-stacking strategy. The performance can be further improved if machine learning is applied to improve the accuracy of weight classification. Yang and Kim (2006) addressed a dynamic version as well as a static version of the location assignment problem. The arrival and retrieval times of the containers were given in the static version, which is described with a mathematical model and solved with a genetic algorithm. For the dynamic problem with uncertain arrival times, heuristic rules for determining the storage location were proposed. Park et al. (2011) proposed an online search algorithm which can dynamically adjust and optimize the stacking policy. Unlike the offline methods which compute the optimal solution before taking any action, the online algorithm continuously generates and evaluates variants of the stacking plan during operation. This is a good option to have a fast reaction to the dynamic setting. Chen and $\mathrm{Lu}$ (2012) addressed the similar problem combining both the space allocation stage and the location assignment stage. The first stage was solved with a mixed integer programming model considering the travelling distance and imbalance of workload. The detailed locations were solved in the second stage with a hybrid sequence stacking algorithm. Both stages were solved under a static case without considering the uncertainties.

\subsection{Re-Marshalling}

Even when the slots for each container are carefully planned, they can still be stacked in the wrong order upon their arrival, due to the lack of accurate information or for other reasons (Steenken et al., 2004). One solution is to pre-marshal the containers according to the retrieval requirements. Lee and Hsu (2007) studied the pre-marshalling problem for a single bay to minimize the number of container movements during the pre-marshalling process. The problem is treated as a multi-commodity flow problem and formulated as an integer programming model. The similar topic was studied in Lee and Chao (2009) for much larger scale problems with a neighborhood search algorithm. Kim and Bae (1998) studied the pre-marshalling of export containers among several bays. In order to reduce the reshuffles, the current bay layout needs to be converted into a desirable bay layout. They used the hierarchical approach to de- compose the problem into three sub-problems. Firstly, choose the target bays from the current bays to match with the required layout. Secondly, decide the amount of containers to be moved from a specific bay to the target bays. Finally, determine the task sequencing of container moves to minimize the completion time of remarshaling. The first and final sub-problems are solved with dynamic programming, while the second is solved as a transportation problem. Although this paper studied the pre-marshalling problem, the detailed relocation of individual containers was not considered. Choe et al. (2011) further studied the pre-marshalling between bays considering the detailed locations of individual containers. A good target stacking configuration was generated with a simulated annealing algorithm, which was then evaluated with the crane working schedule for moving the containers.

Another solution is to reduce the future reshuffles which are caused by the re-marshalling of containers on top of a requested one. Kim and Hong (2006) studied the re-marshalling of containers during the container pickup process. The re-marshallings occur only at the moment of retrieving a target container, and the relocated containers may cause additional future re-marshallings if the position is not well chosen. The objective is to reduce the expected additional re-marshallings where both the stacking configuration of all containers in the bay and the retrieval precedence of containers are given. They proposed a heuristic method to find solutions close to the optimum, while the computation time was much shorter than the branch and bound algorithm and suitable for real-time decisions. Wan et al. (2009) studied the re-marshalling of export containers within a stack to minimize the total amount of reshuffles. An integer programming model was formulated for a static problem to retrieve all the containers within a stack when there are no incoming containers. An IP-based heuristic was proposed to find the solution and the heuristic was then extended to the dynamic setting where there are continual incoming and outgoing containers. Lee and Lee (2010) proposed a three-phase heuristic to solve for an optimal plan of container retrieval. The main objective was to minimize the weighted sum of the container movements and the crane working time. The numerical results showed that the number of movements in the final plan was close to the lower bound, and the heuristic can solve instances of real scale problem. Caserta et al. (2011) considered a dynamic programming approach by transforming the container re-marshalling problem into a shortest path problem. A corridor method was proposed to shorten the search tree and accelerate the search process. Caserta et al. (2012) further studied the same problem and prove the NP-hardness of the container re-marshalling problem. Two different binary integer formulations were proposed. The first maps the complete feasible region of the problem leading to large search space, while the second formulation decreases 
the feasible region with realistic assumptions and is more useful in application.

\section{SUMMARY AND FUTURE RESEARCH CHALLENGES}

This paper gives a comprehensive review for the studies on the yard management in container terminals. From three aspects, i.e., yard cranes management, yard vehicles management, and yard spaces management, this paper reports the advances in these three areas. These research areas are the typical topics among the yard management related papers. They cover the decision problems of main resources involved in the yard management. It may be not easy to establish a totally new research area besides the above three areas in the near future. Instead of extending researches on the width dimension, researches could further improve the existing studies on the depth dimension, which means the models should be formulated with more comprehensive and detailed considerations, such as uncertainty issues, traffic congestion issues, land utilization issues, new features of automated yard systems. The purpose of this paper is to stimulate more practically relevant research in this emerging area. Some future directions on the yard management researches are listed as follows.

\section{(1) Yard management with considering the yard traffic congestion}

Traffic congestion is one of the most important causes that limit a port's efficiency, e.g., the port's throughput rate. The traffic congestions can be the situations that plenty of yard vehicles are waiting for discharging/ loading containers in a small area under a yard crane or a quay crane; the traffic congestions can also be situations that yard vehicles slow and even stop along a lane when some vehicles run along a lane simultaneously. Due to the complexity of modeling the traffic congestions, few studies investigate the yard traffic congestion and obtain some methodologies for modeling the traffic congestion explicitly. Based on the traffic congestion models, the existing studies on yard vehicle dispatching, yard space management, etc. can be extended by considering the yard traffic congestion factors.

\section{(2) Yard management to increase land utilization}

With the growing container traffic, more and more containers will be handled and temporarily stored in the ports. Simple physical expansion of a port is often constrained by the scarcity of land, especially for ports located in or near urban areas, such as Singapore and Shanghai. To meet the growing demands, port operators need sustainable solutions and we are beginning to see more port operators have the intention to adopt automated container terminals (ACTs) with green considerations. However, new ACTs often requires large amount of investment, and the operation efficiency of new ACTs is still an open question. Improvements on the existing systems can be a more direct way to increase the container handling capacity of the limited storage area, while retaining operation efficiency.

\section{(3) Yard management under new designs of port sys- tems}

Nowadays, more and more new designs of ACT systems emerge so as increase the efficiency of port operations, the utilization of land space, and also reduce the labor cost. For example, frame bridge and frame trolley based ACT system, grid-based ACT system, etc. Besides the ACT systems, the traditional design of container terminals is also changing and contains more and more new features, for example, two-layers based terminals, indented berths based terminals, etc. For these new designs of port systems, including the ACT systems, the mathematical models in the existing studies on yard equipments, vehicle, and space management can be further improved or even totally reformulated when considering these new features of the port systems.

\section{(4) Yard management under uncertainties}

Most studies on the yard management decisions are based on a deterministic environment with respect to time and numbers of containers that will be handled. However, the global maritime logistic market contains a lot of uncertainties that inherit from the fluctuation of the demand for freight transportation, and a lot of unforeseen events happening during vessels' voyages. Shipping liners' vessels visit a port periodically (weekly, tendays, or biweekly). For a vessel, the time and the numbers of containers handled are different in each period and also fluctuate along the time. The randomness contained in the uncertain maritime market has brought new challenges for making all kinds of robust yard management decisions so as to improve the efficiency of port operations.

\section{Acknowledgments}

This research is supported by The Program for Professor of Special Appointment (Eastern Scholar) at Shanghai Institutions of Higher Learning, Shanghai ShuGuang project (Grant No. 11SG40), the Fellowship of Container Supply Chain Engineering Research Center, and the Fellowship of University Young Teacher Supporting Program in Shanghai (Grant No. shu11006).

\section{REFERENCES}

Angeloudis, P. and Bell, M. G. (2010), An uncertaintyaware AGV assignment algorithm for automated container terminals, Transportation Research Part E: Logistics and Transportation Review, 46(3), 354- 
366.

Bae, H. Y., Choe, R., Park, T., and Ryu, K. R. (2011), Comparison of operations of AGVs and ALVs in an automated container terminal, Journal of Intelligent Manufacturing, 22(3), 413-426.

Bazzazi, M., Safaei, N., and Javadian, N. (2009), A genetic algorithm to solve the storage space allocation problem in a container terminal, Computers and Industrial Engineering, 56(1), 44-52.

Bish, E. K. (2003), A multiple-crane-constrained scheduling problem in a container terminal, European Journal of Operational Research, 144(1), 83-107.

Bish, E. K., Chen, F. Y., Leong, Y. T., Nelson, B. L., Ng, J. W. C., and Simchi-Levi, D. (2005), Dispatching vehicles in a mega container terminal, $O R$ Spectrum, 27, 491-506.

Bish, E. K., Leong, T. Y., Li, C. L., Ng, J.W., and Simchi-Levi, D. (2001), Analysis of a new vehicle scheduling and location problem, Naval Research Logistics, 48(5), 363-385.

Briskorn, D., Drexl, A., and Hartmann, S. (2006), Inventory-based dispatching of automated guided vehicles on container terminals, OR Spectrum, 28(4), 611-630.

Cao, J. X., Lee, D. H., Chen, J. H., and Shi, Q. (2010), The integrated yard truck and yard crane scheduling problem: Benders' decomposition-based methods. Transportation Research Part E: Logistics and Transportation Review, 46(3), 344-353.

Cao, Z., Lee, D. H., and Meng, Q. (2006a), Scheduling of multiple yard crane system with container loading sequence consideration, Proceedings of the Transportation Research Board 85th Annual Meeting, Washington, DC.

Cao, Z., Lee, D. H., and Meng, Q. (2006b), Scheduling two-transtainer systems for the loading operation of containers using a revised genetic algorithm, Proceedings of the Transportation Research Board 85th Annual Meeting, Washington, DC.

Cao, Z., Lee, D. H., and Meng, Q. (2008), Deployment strategies of double-rail-mounted gantry crane systems for loading outbound containers in container terminals, International Journal of Production Economics, 115(1), 221-228.

Caserta, M., Schwarze, S., and Voß, S. (2012), A mathematical formulation and complexity considerations for the blocks relocation problem, European Journal of Operational Research, 219(1), 96-104.

Caserta, M., Voß, S., and Sniedovich, M. (2011), Applying the corridor method to a blocks relocation problem, OR Spectrum, 33(4), 915-929.

Chang, D., Jiang, Z., Yan, W., and He, J. (2011), Developing a dynamic rolling-horizon decision strategy for yard crane scheduling, Advanced Engineering Informatics, 25(3), 485-494.
Chen, L. and Lu, Z. (2012), The storage location assignment problem for outbound containers in a maritime terminal, International Journal of Production Economics, 135(1), 73-80.

Chen, L., Bostel, N., Dejax, P., Cai, J., and Xi, L. (2007), A tabu search algorithm for the integrated scheduling problem of container handling systems in a maritime terminal, European Journal of Operational Research, 181(1), 40-58.

Chen, L., Langevin, A., and Lu, Z. (2013), Integrated scheduling of crane handling and truck transportation in a maritime container terminal, European Journal of Operational Research, 225(1), 142-152.

Chen, T. (1999), Yard operations in the container terminal: a study in the 'unproductive moves', Maritime Policy and Management, 26(1), 27-38.

Cheung, R. K., Li, C. L., and Lin, W. (2002), Inter block crane deployment in container terminals, Transportation Science, 36(1), 79-93.

Choe, R., Park, T., Oh, M. S., Kang, J., and Ryu, K. R. (2011), Generating a rehandling-free intra-block remarshaling plan for an automated container yard, Journal of Intelligent Manufacturing, 22(2), 201217.

De Castilho, B. and Daganzo, C. F. (1993), Handling strategies for import containers at marine terminals, Transportation Research Part B: Methodological, 27(2), 151-166.

Dekker, R., Voogd, P., and Asperen, E. (2006), Advanced methods for container stacking, OR Spectrum, 28(4), 563-586.

Dorndorf, U. and Schneider, F. (2010), Scheduling automated triple cross-over stacking cranes in a container yard, OR Spectrum, 32(3), 617-632.

Duinkerken, M. B., Dekker, R., Kurstjens, S. T. G. L., Ottjes, J. A., and Dellaert, N. P. (2006), Comparing transportation systems for inter-terminal transport at the Maasvlakte container terminals, OR Spectrum, 28(4), 469-493.

Evers, J. J. and Koppers, S. A. J. (1996), Automated guided vehicle traffic control at a container terminal, Transportation Research Part A: Policy and Practice, 30(1), 21-34.

Gademann, A. J. R. M. and van de Velde, S. L. (2000), Positioning automated guided vehicles in a loop layout, European Journal of Operational Research, 127(3), 565-573.

Gelareh, S., Merzouki, R., McGinley, K., and Murray, R. (2013), Scheduling of intelligent and autonomous vehicles under pairing/unpairing collaboration strategy in container terminals, Transportation Research Part C: Emerging Technologies, 33, 1-21.

Grunow, M., Günther H. O., and Lehmann, M. (2004), Dispatching multi-load AGVs in highly automated seaport container terminals, OR Spectrum, 26(2), 


\section{1-235.}

Grunow, M., Günther H. O., and Lehmann, M. (2006), Strategies for dispatching AGVs at automated seaport container terminals, OR Spectrum, 28(4), 587610.

Guo, X. and Huang, S. Y. (2012), Dynamic space and time partitioning for yard crane workload management in container terminals, Transportation Science, 46(1), 134-148.

Han, Y., Lee, L. H., Chew, E. P., and Tan, K. C. (2008), A yard storage strategy for minimizing traffic congestion in a marine container transshipment hub, OR Spectrum, 30(4), 697-720.

He, J., Zhang, W., Huang, Y., and Yan, W. (2013), A simulation optimization method for internal trucks sharing assignment among multiple container terminals, Advanced Engineering Informatics, 27(4), 598-614.

Hoshino, S., Ota, J., Shinozaki, A., and Hashimoto, H. (2007), Hybrid design methodology and cost-effectiveness evaluation of AGV Transportation systems, IEEE Transactions on Automation Science Engineering, 4(3), 360-372.

Jiang, X., Chew, E. P., Lee, L. H., and Tan, K. C. (2013), Flexible space-sharing strategy for storage yard management in a transshipment hub port, OR Spectrum, 35(2), 417-439.

Jiang, X., Lee, L. H., Chew, E. P., Tan, K. C., and Han, Y. (2012), A container yard storage strategy for improving land utilization and operation efficiency in a transshipment hub port, European Journal of Operational Research, 211(1), 64-73.

Jung, D. H., Park, Y. M., Lee, B. K., Kim, K. H., and Ryu, K. R. (2006), A quay crane scheduling method considering interference of yard cranes in container terminals, In: MICAI 2006: Advances in Artificial Intelligence, Springer, Heidelberg, Germany, 461-471.

Jung, S. H. and Kim, K. H. (2006), Load scheduling for multiple quay cranes in port container terminals, Journal of Intelligent Manufacturing, 17(4), 479492.

Kang, J., Ryu, K. R., and Kim, K. H. (2006), Deriving stacking strategies for export containers with uncertain weight information, Journal of Intelligent Manufacturing, 17(4), 399-410.

Kim, K. H. (1997), Evaluation of the number of rehandles in container yards, Computers and Industrial Engineering, 32(4), 701-711.

Kim, K. H. and Bae, J. W. (1998), Re-marshaling export containers in port container terminals, Computers and Industrial Engineering, 35(3), 655-658.

Kim, K. H. and Bae, J. W. (1999), A dispatching method for automated guided vehicles to minimize delays of containership operations, International Jo- urnal of Management Science, 5(1), 1-25.

Kim, K. H. and Bae, J. W. (2004), A look-ahead dispatching method for automated guided vehicles in automated port container terminals, Transportation Science, 38(2), 224-234.

Kim, K. H. and Hong, G. P. (2006), A heuristic rule for relocating blocks, Computers and Operations Research, 33(4), 940-954.

Kim, K. H. and Kim, H. B. (1999), Segregating space allocation models for container inventories in port container terminals, International Journal of Production Economics, 59(1), 415-423.

Kim, K. H. and Kim, H. B. (2002), The optimal sizing of the storage space and handling facilities for import containers, Transportation Research Part B: Methodological, 36(9), 821-835.

Kim, K. H. and Kim, K. Y. (1999a), Routing straddle carriers for the loading operation of containers using a beam search algorithm, Computers and Industrial Engineering, 36(1), 109-136.

Kim, K. H. and Kim, K. Y. (1999b), An optimal routing algorithm for a transfer crane in port container terminals, Transportation Science, 33(1), 17-33.

Kim, K. H. and Park, K. T. (2003), A note on a dynamic space-allocation method for outbound containers, European Journal of Operational Research, 148(1), 92-101.

Kim, K. H., Jeon, S. M., and Ryu, K. R. (2006), Deadlock prevention for automated guided vehicles in automated container terminals, OR Spectrum, 28(4), 659-679.

Kim, K. H., Kang, J. S., and Ryu, K. R. (2004), A beam search algorithm for the load sequencing of outbound containers in port container terminals, $O R$ Spectrum, 26(1), 93-116.

Kim, K. H., Lee, K. M., and Hwang, H. (2003), Sequencing delivery and receiving operations for yard cranes in port container terminals, International Journal of Production Economics, 84(3), 283-292.

Kim, K. H., Park, Y. M., and Ryu, K. R. (2000), Deriving decision rules to locate export containers in container yards, European Journal of Operational Research, 124(1), 89-101.

Kim, K. Y. and Kim, K. H. (2003), Heuristic algorithms for routing yard-side equipment for minimizing loading times in container terminals, Naval Research Logistics, 50(5), 498-514.

Koo, P. H., Lee, W. S., and Jang, D. W. (2004), Fleet sizing and vehicle routing for container transportation in a static environment, OR Spectrum, 26(2), 193-209.

Lau, H. Y., Wong, V. W., and Lee, I. S. K. (2007), Immunity-based autonomous guided vehicles control, Applied Soft Computing, 7(1), 41-57.

Lee, D. H., Cao, J. X., Shi, Q., and Chen, J. H. (2009), 
A heuristic algorithm for yard truck scheduling and storage allocation problems, Transportation Research Part E: Logistics and Transportation Review, 45(5), 810-820.

Lee, D. H., Cao, Z., and Meng, Q. (2007), Scheduling of two-transtainer systems for loading outbound containers in port container terminals with simulated annealing algorithm, International Journal of Production Economics, 107(1), 115-124.

Lee, Y. and Chao, S. L. (2009), A neighborhood search heuristic for pre-marshalling export containers, European Journal of Operational Research, 196(2), 468-475.

Lee, Y. and Hsu, N. Y. (2007), An optimization model for the container pre-marshalling problem, Сотрuters and Operations Research, 34(11), 3295-3313.

Lee, Y. and Lee, Y. J. (2010), A heuristic for retrieving containers from a yard, Computers and Operations Research, 37(6), 1139-1147.

Lehmann, M., Grunow, M., and Günther H. O. (2006), Deadlock handling for real-time control of AGVs at automated container terminals, OR Spectrum, 28 (4), 631-657.

Li, C. L. and Vairaktarakis, G. L. (2004), Loading and unloading operations in container terminals, IIE Transactions, 36(4), 287-297.

Lim, A. and $\mathrm{Xu}, \mathrm{Z}$. (2006), A critical-shaking neighborhood search for the yard allocation problem, European Journal of Operational Research, 174(2), 12471259.

Lim, J. K., Kim, K. H., Yoshimoto, K., Lee, J. H., and Takahashi, T. (2003), A dispatching method for automated guided vehicles by using a bidding concept, OR Spectrum, 25(1), 25-44.

Linn, R. J. and Zhang, C. (2003), A heuristic for dynamic yard crane deployment in a container terminal, IIE Transactions, 35(2), 161-174.

Linn, R., Liu, J. Y., Wan, Y. W., Zhang, C., and Murty, K. G. (2003), Rubber tired gantry crane deployment for container yard operation, Computers and Industrial Engineering, 45(3), 429-442.

Liu, C. I., Jula, H., Vukadinovic, K., and Ioannou, P. (2004), Automated guided vehicles system for two container yard layouts, Transportation Research Part C: Emerging Technologies, 12(5), 349-368.

Narasimhan, A. and Palekar, U. S. (2002), Analysis and algorithms for the transtainer routing problem in container port operations, Transportation Science, 36(1), 63-78.

Ng, W. C. (2005), Crane scheduling in container yards with inter-crane interference, European Journal of Operational Research, 164(1), 64-78.

Ng, W. C. and Mak, K. L. (2004), Sequencing of container pickup trucks in container yard blocks, Interational Journal of Industrial Engineering, 11(1),
82-89.

Ng, W. C. and Mak, K. L. (2005a), An effective heuristic for scheduling a yard crane to handle jobs with different ready times, Engineering Optimization, 37(8), 867-877.

Ng, W. C. and Mak, K. L. (2005b), Yard crane scheduling in port container terminals, Applied Mathematical Modeling, 29(3), 263-276.

Ng, W. C., Mak, K. L., and Zhang Y. X. (2007), Scheduling trucks in container terminals using a genetic algorithm, Engineering Optimization, 39(1), 33-47.

Nguyen, V. D. and Kim, K. H. (2009), A dispatching method for automated lifting vehicles in automated port container terminals, Computers and Industrial Engineering, 56(3), 1002-1020.

Nishimura, E., Imai, A., and Papadimitriou, S. (2005), Yard trailer routing at a maritime container terminal, Transportation Research Part E: Logistics and Transportation Review, 41(1), 53-76.

Park, T., Choe, R., Kim, Y. H., and Ryu, K. R. (2011), Dynamic adjustment of container stacking policy in an automated container terminal, International Journal of Production Economics, 133(1), 385-392.

Rajeeva, L. M., Wee, H. G., Ng, W. C., and Teo, C. P. (2003), Cyclic deadlock prediction and avoidance for zone-controlled AGV system, International Journal of Production Economics, 83(3), 309-324.

Reveliotis, S. A. (2000), Conflict resolution in AGV systems, IIE Transactions, 32(2), 647-659.

Sauri, S. and Martin, E. (2011), Space allocating strategies for improving import yard performance at marine terminals, Transportation Research Part E: Logistics and Transportation Review, 47(6), 10381057.

Sculli, D. and Hui, C. F. (1988), Three dimensional stacking of containers, Omega, 16(6), 585-594.

Steenken, D. (1992), Fahrwegoptimierung am containerterminal under echtzeitbedingungen, OR Spektrum, 14(3), 161-168.

Steenken, D., Henning, A., Freigang, S., and Vo $\beta$, S. (1993), Routing of straddle carriers at a container terminal with the special aspect of internal moves, OR Spectrum, 15(3), 167-172.

Steenken, D., Vo $\beta$, S., and Stahlbock, R. (2004), Container terminal operation and operations research: a classification and literature review, OR Spectrum, 26(1), 3-49.

Taleb-Ibrahimi, M., de Castilho, B., and Daganzo, C. F. (1993), Storage space vs handling work in container terminals, Transportation Research Part B: Methodological, 27(1), 13-32.

Vis, I. F. and Carlo, H. J. (2010), sequencing two cooperating automated stacking cranes in a container terminal, Transportation Science, 44(2), 169-182.

Vis, I. F. and Harika, I. (2004), Comparison of vehicle 
types at an automated container terminal, OR Spectrum, 26(1), 117-143.

Vis, I. F., de Koster, R., Roodbergen, K. J., and Peeters, L. W. (2001), Determination of the number of automated guided vehicles required at a semiautomated container terminal, Journal of the $\mathrm{Op}$ erational Research Society, 52(4), 409-417.

Wan, Y. W., Liu, J., and Tsai, P. C. (2009), The assignment of storage locations to containers for a container stack, Naval Research Logistics, 56(8), 699713.

Watanabe, I. (1991), Characteristics and analysis method of efficiencies of container terminal: an approach to the optimal loading/unloading method, Container Age, 3, 36-47.

Wu, Y., Luo, J., Zhang, D., and Dong, M. (2013), An integrated programming model for storage management and vehicle scheduling at container terminals, Research in Transportation Economics, 42(1), 13-27.

Xue, Z., Zhang, C., Miao, L., and Lin, W. H. (2013), An ant colony algorithm for yard truck scheduling and yard location assignment problems with precedence constraints, Journal of Systems Science and Systems Engineering, 22(1), 21-37.

Yang, C. H., Choi, Y. S., and Ha, T. Y. (2004), Simulation-based performance evaluation of transport vehicles at automated container terminals, OR Spectrum, 26(2), 149-170.
Yang, J. H. and Kim, K. H. (2006), A grouped storage method for minimizing relocations in block stacking systems, Journal of Intelligent Manufacturing, 17(4), 453-463.

Zhang, C., Liu, J., Wan, Y. W., Murty, K. G., and Linn, R. J. (2003), Storage space allocation in container terminals, Transportation Research Part B: Methodological, 37(10), 883-903.

Zhang, C., Wan, Y. W., Liu, J., and Linn, R. J. (2002), Dynamic crane deployment in container storage yards, Transportation Research Part B: Methodological, 36(6), 537-555.

Zhang, L. W., Ye, R., Huang, S. Y., and Hsu, W. J. (2005), Mixed integer programming models for dispatching vehicles at a container terminal, Journal of Applied Mathematical Computing, 17(1-2), 145-170.

Zhen, L. (2013), Yard template planning in transshipment hubs under uncertain berthing time and position, Journal of the Operational Research Society, 64, 1418-1428.

Zhen, L., Chew, E. P., and Lee, L. H. (2011), An integrated model for berth template and yard template planning in transshipment hubs, Transportation Science, 45(4), 483-504.

Zyngiridis, I. (2005), Optimizing container movements using one and two automated stacking cranes, Master's thesis, Naval Postgraduate School, Monterey, CA. 